\title{
O cuidado da população em situação de vulnerabilidade: atuação e desafios enfrentados por uma equipe do consultório na rua em uma cidade do interior do
}

\section{Maranhão}

\author{
Taking care of the population in vulnerable situation: performance and challenges faced by a team \\ of the office on the street in a city of the interior of Maranhão \\ Cuidado de la población en situación de vulnerabilidad: desempeño y desafíos enfrentados por un \\ equipo de la oficina en la calle en una ciudad del interior de Maranhão
}

Recebido: 23/11/2021 | Revisado: 29/11/2021 | Aceito: 02/12/2021 | Publicado: 12/12/2021

Willian de Sousa Ramos
ORCID: https://orcid.org/0000-0002-5926-4698
Universidade CEUMA, Brasil
E-mail: willyaan-sr@ hotmail.com
Haigle Reckziegel de Sousa
ORCID: https://orcid.org/0000-0002-5803-2289
Universidade CEUMA, Brasil
E-mail: haiglereck3@ @mail.com
Thaís Rodrigues de Sousa Silva
ORCID: https://orcid.org/0000-0002-7798-4461
Universidade CEUMA, Brasil
E-mail: thais_itz@ hotmail.com
Cristina Limeira Leite
ORCID: https://orcid.org/0000-0002-7321-1496
Universidade CEUMA, Brasil
E-mail:crislimeira@gmail.com
Patrícia dos Santos Silva Queiroz
ORCID: https://orcid.org/0000-0002-9587-1786
Universidade CEUMA, Brasil
E-mail:patrícia.queiroz@ceuma.br

\section{Resumo}

Objetivo: compreender como atua a equipe do consultório na rua e quais os desafios enfrentados na manutenção da saúde da população em situação de rua. Método: estudo qualitativo de caráter exploratório e descritivo, realizado com profissionais da equipe, por meio de entrevista, em junho de 2021. Resultados: A equipe atua prestando assistência de acordo com os princípios basilares do Sistema Único de Saúde (SUS) e da Política Nacional para a População em Situação de Rua. Ainda através de meios que colaboram na assistência, incluindo a redução dos danos como estratégia, existe a ação de escuta ativa com o acolhimento e a articulação da rede. Considerações finais: $\mathrm{O}$ estudo mostra que os profissionais da equipe possuem conhecimento sobre a atuação e finalidade do consultório na rua, demonstrando a importância desta equipe para a população em situação de vulnerabilidade. Observou-se também as articulações com a rede de saúde para prestação de assistência continuada com objetivo de reduzir os danos.

Palavras-chave: Pessoas em situação de rua; Redução do dano; Atenção à saúde; Enfermagem em Saúde Pública.

\begin{abstract}
Objective: to understand how the office team works on the street and what challenges are faced in maintaining the health of the homeless population. Method: qualitative exploratory and descriptive study, carried out with team professionals, through interviews, in June 2021. Results: The team works by providing assistance in accordance with the basic principles of the Unified Health System (SUS) and the National Policy for the Homeless Population. Also through means that collaborate in care, including harm reduction as a strategy, there is an active listening action with the reception and articulation of the network. Final considerations: Through the study, it was found that the team professionals have knowledge about the performance and purpose of the office on the street, demonstrating the importance of this team for the population in a vulnerable situation. It was also observed the articulations with the health network to provide continued care with the objective of reducing damage.
\end{abstract}

Keywords: Homeless persons; Harm reduction; Delivery of health care; Public Health Nursing. 


\section{Resumen}

Objetivo: comprender cómo trabaja el equipo de oficina en la calle y qué desafíos se enfrentan para mantener la salud de la población sin hogar. Método: estudio cualitativo, exploratorio y descriptivo, realizado con profesionales del equipo, mediante entrevistas, en junio de 2021. Resultados: El equipo trabaja brindando asistencia de acuerdo con los principios básicos del Sistema Único de Salud (SUS) y la Política Nacional de Población sin Hogar. También a través de los medios que colaboran en la atención, incluyendo la reducción de daños como estrategia, existe una acción de escucha activa con la recepción y articulación de la red. Consideraciones finales: A través del estudio, se encontró que los profesionales del equipo tienen conocimiento sobre el desempeño y propósito de la oficina en la calle, demostrando la importancia de este equipo para la población en situación de vulnerabilidad. También se observó las articulaciones con la red de salud para brindar atención continua con el objetivo de reducir los daños.

Palabras clave: Personas sin hogar; Reducción del daño; Atención a la salud; Enfermería en Salud Pública.

\section{Introdução}

O habitar na rua consiste em um fenômeno social que desencadeia um problema de saúde pública como consequências das desigualdades sociais em vários países sejam os desenvolvidos, os em desenvolvimento e subdesenvolvidos. O contexto que leva a situação de rua é vivências singulares a até traumáticas desde rupturas familiares, abuso de substâncias psicoativas, violência sexual, física e emocional, maus tratos e buscas malsucedidas por melhores condições de sobrevivência. (Bittencourt, et al. 2019)

Devido a isso, pessoas em situação de rua estão constantemente vulneráveis e expostas. Sendo assim destituídas de direitos fundamentais e sociais como acesso a saúde, a moradia, educação, lazer, trabalho, dentre outros direitos, assim colaborando para as discrepâncias sociais em saúde. (Oliveira, et al., 2018)

Visando aumentar a acessibilidade dos serviços de saúde para a população em situação de rua, foram definidas as diretrizes de organização e funcionamento das equipes de consultório na rua (eCR), através da Portaria $\mathrm{N}^{\circ} 122$, de 25 janeiro de 2011, instituída pelo Ministério da Saúde considerando a Política Nacional para a População em Situação de Rua e o Comitê Técnico de Saúde para a população em situação de rua, e em conformidades com a nova atualização através da Portaria $\mathrm{N}^{\circ} 1.255$ de 18 de junho de 2021 que dispõe sobre as diretrizes de organização e funcionamento das equipes de Consultório na Rua e os critérios de cálculo do número máximo de equipes de Consultório na Rua, por município e Distrito Federal, por meio da alteração da Portaria de Consolidação GM/MS no 2, de 28 de setembro de 2017. O Consultório de Rua consiste em uma equipe multiprofissional, tendo suas ações em conformidades com a Política Nacional de Atenção Básica. (Brasil, 2021)

Ainda conforme a portaria, prevê a composição e a organização da equipe de Consultório de Rua (eCR), sendo constituída pelos seguintes profissionais: enfermeiro, psicólogo, médico, terapeuta ocupacional, assistente social, agente social, técnico ou auxiliar de enfermagem e o técnico em saúde bucal. Organizada em formato de modalidade I, II e III: a modalidade I pode ser composta por dois profissionais do ensino superior e dois profissionais do ensino médio, já a modalidade II é composta por três profissionais do ensino superior e três profissionais do ensino médio, a modalidade III segue a mesma composição da modalidade II, sendo acrescentada a participação do profissional médico. (Brasil, 2012)

As ações dos Consultórios de Rua são realizadas em conformidade com suas diretrizes e princípios, estes estão descritos no Decreto $N^{\circ} 7053$ que institui a Política Nacional para a População em Situação de Rua. Segundo este decreto dentre os princípios está o atendimento humanizado e universalizado, além do respeito aos fatores distintos como raça, idade, gênero. Os objetivos da política direcionam as ações da equipe Consultório de Rua, tais como acessibilidade integral aos serviços de saúde, promover educação permanente dos profissionais que compõem a equipe, levantamento do número da população em situação de rua, proporcionar o acesso aos benefícios assistenciais, previdenciários e segurança alimentar. (Brasil, 2009)

O Brasil apresenta falha na coleta de dados e no número fidedigno de moradores de rua, segundo a literatura, o Brasil 
tem déficit dessas informações, como resposta, é relatada a dificuldade de realizar uma pesquisa de campo ampla com uma população que não possui endereço fixo. Tal falta de interesse na coleta de dados, acarreta na ausência de informações de pessoas vulneráveis, o que ocasiona no esquecimento e na dificuldade de acessibilidade dessa população aos serviços e benefícios sociais garantidos pelo governo. (Lima, et al., 2019)

De acordo com o Instituto de Pesquisa Econômica Aplicada (IPEA) no Texto para Discussão Estimativa da População em Situação de Rua no Brasil, apontou que no país, no ano de 2015, existia um total de 101.854 moradores de rua, que o número maior foi constatado na cidade de São Paulo com um montante de 15.905 pessoas em situação de rua. (IPEA, 2016)

Para a assistência e manutenção do cuidado da população em situação de rua é necessário à busca ativa desses moradores, sendo um desafio no campo da saúde coletiva para a equipe. Dessa mesma forma, existe a curiosidade de conhecer e relatar a atuação da equipe do Consultório na Rua no Município de Imperatriz/MA. Portanto, a principal contribuição deste estudo consiste na reflexão sobre a prática assistencial junto a esta população, para construção de estratégias que possam minimizar os danos e garantir a proteção e o cuidado integral.

Diante disso, como a equipe do Consultório na Rua atua conforme política realizando os cuidados para as pessoas em situação de rua, as seguintes questões nortearam a pesquisa: Como atuam as eCR? Que desafios enfrentam para realizar o atendimento a essa população? Quais os determinantes sociais e ambientais que interferem na manutenção da saúde dessa população?

Portanto, o presente estudo tem por objetivo compreender como atua a equipe do consultório na rua e quais os desafios enfrentados na manutenção da saúde dessa população na cidade de Imperatriz/MA.

\section{Metodologia}

\section{Aspectos éticos}

Essa pesquisa atendeu aos preceitos éticos constantes na Resolução n. 510/2016, do Conselho Nacional de Saúde, obtendo parecer favorável do Comitê de Ética e Pesquisa com Seres Humanos da Universidade Ceuma, Parecer CEP $n^{\circ}$ 4.696.472 CAAE: 42284621.3.0000.5084.

\section{Referencial teórico-metodológico e tipo de estudo}

Trata-se de um estudo de natureza quantitativa e qualitativa de caráter exploratório e descritivo, tendo a compreensão da atuação da equipe do consultório na rua e quais os desafios enfrentados na manutenção da saúde dessa população vulnerável o objetivo principal deste estudo. Uma pesquisa exploratória cria-se certa aproximação com o objeto de investigação. Segundo o autor, pode-se afirmar que a maioria das pesquisas realizadas com propósitos acadêmicos, pelo menos num primeiro momento, assume o caráter de pesquisa exploratória, pois neste momento é pouco provável que o pesquisador tenha uma definição clara do que irá investigar. (Gil, 2016)

A pesquisa qualitativa é um tipo de investigação voltado para as características qualitativas do fenômeno estudado, considerando a parte subjetiva do problema. Ela se preocupa com aspectos da realidade que não podem ser quantificados, centrando-se na compreensão e na explicação da dinâmica das relações sociais. (Lozada, 2018)

\section{Cenário do estudo e participantes}

De acordo com os dados coletados através do Instituto Brasileiro de Geografia e Estatísticas - IBGE, a cidade de Imperatriz tem uma estimativa para o ano de 2020 de 259.980 habitantes. Estima-se que a taxa de desempregados em Imperatriz do Maranhão segundo o Cadastro Geral de Empregados e Desempregados (CAGED) é de 12 mil pessoas. 
Atualmente, esse número sofreu um sobressalto em todo o mundo, devido a Pandemia causada pelo novo Coronavírus. (IBGE, 2021)

O Consultório na Rua da Cidade de Imperatriz/MA iniciou suas atividades no ano de 2011, está situado na Rua Dom Pedro I, no bairro Beira Rio, atua fazendo a busca ativa por toda a cidade, o que dificulta identificar sua área de abrangência.

O estudo foi realizado com a Equipe do Consultório na Rua, do município de Imperatriz/MA que atualmente conta com uma equipe multiprofissional composta por médico, enfermeira, assistente social, agente social, técnico de enfermagem, técnico em saúde bucal e psicólogo, consolidando-se na modalidade III.

\section{Coleta e organização dos dados}

Os dados foram coletados no mês de junho de 2021, após aprovação do Comitê de Ética e Pesquisa, Parecer CEP no 4.696.472 CAAE: 42284621.3.0000.5084.

Para viabilizar o primeiro contato com a eCR, foi combinado dia e horário compatível com o turno de trabalho da equipe, neste caso o turno noturno. Na oportunidade, foi possível conhecer os profissionais que atuam na eCR, apresentar o estudo e realizar o convite individual para participarem. Após o primeiro contato, os pesquisadores agendaram datas para coleta de dados com os profissionais.

As entrevistas foram conduzidas a partir dos seguintes questionamentos: Há quanto tempo trabalha no Consultório na Rua? Quais as atividades realizadas pela equipe do Consultório na Rua? Como é realizado o atendimento a pessoa em situação de rua? Como é sua relação com os moradores de rua? Já teve algum acontecimento ruim na relação com os moradores de rua? Quais os serviços são ofertados pelo Consultório na Rua de Imperatriz? O Consultório na Rua faz articulação com alguma ESF? Como é feito essa parceria? Quais os principais fatores que dificultam a assistência à pessoa em situação de rua? Quais os desafios encontrados no momento de ofertar os serviços de saúde? Cabe ressaltar que, no momento da entrevista, somente estavam presentes o pesquisador e o participante.

Antes de concederem as entrevistas, os participantes foram esclarecidos quanto aos objetivos do estudo e sobre a importância de suas participações na pesquisa. O Termo de Consentimento Livre e Esclarecido (TCLE) foi lido pelos pesquisadores e assinado pelos participantes. No período da coleta de dados, a equipe abordou 6 pessoas que atendiam aos critérios de inclusão, não havendo recusas de participação.

As entrevistas foram realizadas individualmente, em momentos em que os profissionais em se mostravam disponíveis para concederem seus depoimentos. Considerando a necessidade de privacidade com o mínimo de ruídos possível. Os depoimentos foram gravados em áudio e tiveram a duração de aproximadamente 20 minutos cada. Para preservação do anonimato, as participantes foram identificadas com codinomes de flores.

\section{Análise dos dados}

A organização, categorização e análise do material obtido na entrevista, foi analisado pela equipe de pesquisadores. Assim a partir da leitura flutuante de cada depoimento apreciou e assimilou sobre a atuação e desafios enfrentados pela eCR no cuidado, além das formas como a equipe se organiza e as necessidades em saúde da população em situação de rua. Para organização do material utilizando-se da técnica de análise de conteúdo categorial por temática, proposta por Bardin. Após a transcrição das entrevistas, será realizada uma leitura exaustiva e exploração minuciosa do material. Em seguida, far-se-á o tratamento dos resultados, gerando categorias identificadas através dos sentimentos expressados pelos participantes. (Bardin, 2017) 


\section{Resultados}

\section{Caracterização dos participantes}

Participaram do estudo, 6 (100\%) profissionais de saúde (Enfermeira, Coordenadora, Assistente Social, Psicóloga, Técnica de Enfermagem e Médica) membros da equipe, sendo todos os membros do sexo feminino. A faixa etária dos participantes variou entre 25 e 47 anos, sendo a idade média de 36,8 anos. Quanto a escolaridade das entrevistadas, a grande maioria, 5(83,3\%) possuem o Ensino Superior e 1(16,7\%) o Ensino Médio. Em se tratando do estado civil, 4(66,3\%) são casadas e 2(33,3\%) são solteiras. Quanto ao tempo de atuação na equipe, 2(33,3\%) atuam a 2 meses, 1(16,7\%) atua a 2 anos, $2(33,3 \%)$ atuam a 6 anos e $1(16,7 \%)$ atua a oito anos na equipe.

Da análise de conteúdo emergiram quatro categorias temáticas: (1) desafios para prestar assistência; (2) ações realizadas pela equipe; (3) elementos que colaboram com a assistência; (4) fatores que dificultam a assistência continuada.

\section{Categorias que emergiram do estudo \\ Desafios para prestar assistência}

De acordo com os participantes, as dificuldades enfrentadas pela equipe se relacionam com a própria situação de vulnerabilidade dos pacientes atendidos pelo consultório na rua. Os relatos indicam que o atendimento ser na rua sem a condição de ter um local próprio, além da rejeição dos pacientes de permanecer naquele estado e na recusa por tratamento: "a dificuldade maior é a falta [...] como eu diria, a rejeição dele em sair da rua né, e permanecer naquelas condições, naquele local, esse, eu acho que é o entrave maior, porque você tratar né, falar de saúde, pra alguém que não tem o mínimo." (Amarílis)

"tem a dificuldade, essa questão de não poder oferecer tudo na rua, e às vezes eles não querem ir para outro lugar né, não querem sair dali para fazer um exame, não querem sair dali pra, pra ir ao hospital, então as vezes a gente não consegue suprir a necessidade deles ali na rua." (Magnólia)

A dificuldade em prestar assistência ao paciente usuário de drogas segue enfatizada pelos participantes, pois relatam receio e medo de possíveis reações agressivas e questões relacionadas com insegurança:

"Maior o desafio, é porque são pessoas né, usuárias de drogas, e a gente ficam meio com receio, quando eles estão naqueles dias, meio alterado, a gente fica com receio de chegar ate eles, e medo [...] “(Petúnia)

“As drogas, né?! Por que a dificuldade maior do auxílio pra a eles né?!, É... são as drogas porque eles as vezes eles recusam o atendimento, eles recusam a ajuda [...]” (Verbena)

“[...] já teve situações que a gente se sentiu ameaçada né, algum estado, por exemplo, um paciente que estava sob uso de drogas, que ele ficou um pouco mais agitado do que o normal, ai a gente se sentiu ameaçado, a gente vai embora nesses casos, entendeu? A gente começa a consulta, mas se a gente ver que tá tendo ali uma probabilidade de uma, alguma ameaça, a gente já entra na van e já não faz mais o atendimento." (Orquídea)

\section{Ações realizadas pela equipe}

As ações que são realizadas pela equipe relacionam com os serviços de saúde conforme preconizado pela Política Nacional de Atenção Básica, em atender as necessidades do paciente assim configura-se como porta de entrada a atenção psicossocial sendo integrado como atenção primária, como serviço de Unidade Básica de Saúde que se compõe com equipe multidisciplinar: 
"consultas, encaminhamentos, atendimento psicológico, tudo relacionado à assistência, nós também, fora que a gente ainda tem nossos parceiros né, porque nós contamos com uma rede de atenção né, seja ela clinica, seja ela psicossocial, seja ela assistência [...]" (Amarílis)

"prestamos atendimentos dentro do campo da atenção básica, então nós temos medicamentos, né... A oferecer pelo mesmo da farmácia básica, consulta médica, consulta de enfermagem, abordagem psicológica também e acompanhamento da assistente social [...]" (Magnólia)

"A gente realiza os procedimentos, né?! que são as consultas médicas, são administração de medicamentos, a gente realiza palestras também e faz visitas nas comunidades e nas ruas com as pessoas em situação de rua." (Petúnia)

"Serviços, a gente tem a equipe né, toda montada, serviços médicos prestados por mim, tem a enfermeira, que é quem faz curativo, troca o curativo [...] tem a psicóloga né, que, sempre ela faz acompanhamento, que é uma pessoa que realmente é necessária né [...] tem também a agente social né, assistente social [...]” (Orquidea)

\section{Elementos que colaboram com a assistência}

Conforme as falas dos participantes apontam que as ações da equipe são facilitadas por elementos que apoiam e promovem o suporte para prestar cuidados de saúde para os pacientes. Assim, surgem três subcategorias: a) Acolhimento profissional; b) Articulação da rede pela equipe; c) Disponibilidade da equipe.

\section{Acolhimento profissional}

Ao estabelecer acolhimento conforme preconiza a política de humanização. A escuta ativa favorece o acolhimento desde o primeiro contato que pode ser realizado por qualquer profissional dentro da equipe, servindo como ferramenta que facilita a prestação da assistência e dos cuidados:

"Qualquer um que quiser pode descer e fazer primeiramente esse contato, esse acolhimento, então a gente chega e fala é... o que é a equipe, que é uma equipe de saúde, se tá precisando de alguma coisa, como a gente não conhece na primeira vez pra eles não se assustar não desce a equipe toda, depois a equipe vai descendo aos poucos e a gente vai vendo no momento o que aquele paciente tá necessitando." (Verbena)

"Tem aquela conversa e a partir, a gente tem que criar um vinculo primeiro com eles pra eles poderem ter confiança que a gente é profissional, é da saúde, estamos ali para ajuda-los e ai eles começam a conversar com a gente, e ai dependendo da demanda que eles venham a pedir, a necessitar a gente presta esse atendimento." (Magnólia)

"[...] A gente faz a escuta e o acompanhamento dos usuários em situação de rua [...] Dependendo da onde a gente faz o atendimento a gente, a escuta, por exemplo, se a gente estiver num albergue e a pessoa quiser o atendimento do psicólogo à gente consegue um local reservado, na rua a gente só se afasta um pouco das pessoas para fazer essa escuta, porque na rua não tem um local." (Verbena)

\section{Articulação da rede pela equipe}

A articulação da rede visando assim atender ao princípio da integralidade. Essa estratégia norteia as ações no cumprimento do cuidado, reconhecimento nos discursos dos participantes sobre a importância quando realizam encaminhamentos para rede e articulando a integração dos pacientes nos serviços, além do suporte em instituições, como 
setores jurídicos.

"[...] então a gente tem sempre os parceiros da sede, os parceiros da saúde mental, os parceiros da urgência e emergência, então todas essas outras instituições, elas são parceiras do consultório na rua, nós trabalha em conjunto com ele, então todos os serviços que são ofertados na UBS e inclusive que são feitos pelos profissionais dos NASF, o consultório na rua dispõe para as pessoas em situação de rua.” (Amarílis)

“Para outros órgãos, a gente, também faz esse vínculo, com as outras instituições, de explicar que ele tá indo lá, ou então de até leva-lo ao um hospital [...] às vezes a gente vai com ele pra poder ter esse primeiro contato, e eles se sentirem acolhidos [...] também nesse requisito de retirada de documentos, é encaminhamento pra órgãos competentes, Ministério Público, pra abrigos, toda essa questão mesmo social, então a gente é uma equipe [...]” (Magnólia)

"Fora isso a gente encaminha a necessidade de um outro serviço que esteja dentro da rede de atenção à saúde, esse usuário ele é encaminhado [...] para atendimento de uma outra especialidade que seja necessária. Eles são encaminhados dentro da rede.” (Verbena)

\section{Disponibilidade da equipe}

A disponibilidade da equipe que trabalha de forma rotineira mantendo o vínculo com os pacientes.

“É a gente trabalha de segunda a quinta [...] segunda, terça e quarta à noite, de sete até as onzes, isso é o nosso horário fixo, porém as vezes dependendo da demanda a gente pode ficar até quando haja necessidade e nas quintas feiras nas comunidades terapêuticas" (Amarílis)

“A rotina costuma ser de segunda a quinta-feira, e nos dia de quinta-feira a gente vai para os abrigos e durante a noite a gente vai sempre, é, nas ruas mesmo, nas praças, nas ruas [...] Como é diário né nossa visita, todas as noites, é fácil manter contato com eles, a gente vai ter um vínculo com eles. ”(Tulipa)

\section{Fatores que dificultam a assistência continuada}

Nessa categoria referem-se os dilemas de manter a assistência contínua, nas falas dos participantes são apontadas a própria vulnerabilidade dos pacientes de ser usuários de drogas, a rejeição dos mesmos em ser atendidos e o déficit no autocuidado dos usuários:

"Primeiro, porque eles são itinerantes, como eu falei para você, ele não ficam no mesmo lugar [...] é a primeira dificuldade, para você ter um atendimento continuo com um paciente que ele não tem essa frequência que ele é itinerante [...] segunda dificuldade é fazer com que ele aceite o tratamento, um paciente que vive em situação de rua, o intuito dele, a maioria das vezes, ele é, fazer uso abusivo, seja de álcool, seja de droga e, fica difícil pra você acompanhar um paciente desse. " (Amarilis)

"Principalmente as grávidas [...] está em situação de uso de álcool e droga, e a gente tenta também fazer essa medida de tentar diminuir o dano [...] mas não é cento por cento a nossa abordagem, a gente está mesmo ali para ver se está acontecendo um aborto que é uma medida de emergência que elas deixam [...].” (Magnólia) 
"Porque às vezes eles recusam o atendimento por causa da droga [...] E você, e a gente precisa aprender a respeitar, então o trabalho vai muito no foco da redução de danos. De minimizar esse impacto e esse sofrimento. E tentar resgatar. " (Verbena)

“Eles não aceitam ser acompanhados, eles acham que a gente está observando eles, que a gente está perseguindo, que a gente vai chamar a polícia, então o principal fator é esse, a não aceitação deles, eles mesmo que se recusam, às vezes a gente chega perto eles dizem “não, não quero não”, a gente nem chega, esse é o principal fator.” (Orquídea)

\section{Discussão}

Observou-se que os profissionais enfatizam que a próprio perfil dos pacientes gera as dificuldades do atendimento associado por ser realizado na rua propriamente dita, relacionado a isso o receio e a insegurança. Além disso, a própria rejeição do paciente em situação de rua de não querer receber atendimento e não sair daquela condição para um serviço de saúde.

Sobre a atuação da equipe do consultório na rua possui um ambiente de trabalho que difere de outros serviços de saúde, não sendo o convencional da assistência, por ser na rua. Dessa forma, o trabalho no cenário da rua apresenta dificuldades que não intrínsecas a esse espaço associando a isso a recusa do paciente de sair do ambiente para receber atendimentos nas instituições de saúde, assim a equipe realiza sua atuação com limites. Além disso, o espaço e o perfil do paciente em situação de rua geram na equipe um sentimento de risco para a sua integridade. (Rosa, et al. 2016)

Como característica principal do consultório de rua consiste na redução do dano, assim observam-se ações norteadas para isso, como observado pelas falas ações como troca de curativo, consultas, distribuição de medicamento, atendimento com psicólogo e assistente social.

Dentro do contexto de prestação de atendimentos em saúde, a equipe que integra o consultório na rua é multiprofissional e atuam em ações para redução de danos do público que vive nas ruas já em situação vulnerável. Conforme da Política Nacional de Atenção Básica (PNAB) que consolida a atenção à saúde a população em situação de rua a equipe atua em nível de atenção básica sendo a porta de entrada para esse público, assim atuar frente aos diferentes problemas e necessidades de saúde. As ações são assistenciais norteadas pelos princípios e diretrizes do SUS e da Política Nacional para a População em Situação de Rua, que se propõe assim o cuidado integral como ressaltado nas falas, com consultas, encaminhamentos para a rede seguindo a clínica além de atenção ao psicossocial. (Santos, et al., 2021)

Sobre as facilidades os participantes apresentaram o acolhimento assim favorecendo a escuta entre a equipe e os pacientes, além disso, a articulação da rede e a disponibilidade da equipe em estabelecer rotina que mantém o vínculo com os pacientes. Nesse sentido, a identificação no discurso dos participantes sobre a escuta ativa é uma estratégia de humanização do atendimento sendo assim, uma atuação relevante e positiva que está em conformidade com as ações da Política Nacional de Atenção Básica e da Política para a Atenção Integral ao Uso de Álcool e Outras Drogas, ainda das próprias diretrizes para ações da equipe do Consultório na rua. (Engstrom \& Teixeira, 2016)

Nos dados evidenciam-se os participantes a articulação da rede de atenção à saúde como recurso primordial para a assistência aos pacientes. É fundamental essa articulação com a rede, visando o acesso ao espaço físico do serviço de saúde, ou seja, legitimar o direito dos pacientes que vivem em situação de rua sistema de saúde e seus recursos como direito de todos a acessar qualquer serviço de saúde. (Ferreira, et al., 2016)

Nesse sentido, a equipe de consultório na rua conforme Política Nacional de Atenção Básica ainda é incluída na Rede de Atenção Psicossocial como componente da atenção básica se constituindo como o primeiro contato para os serviços de saúde mental. (Pinto, et al., 2018)

A atuação da equipe que cumpre com trabalho diário mantendo a disponibilidade com ações pela manutenção do 
vínculo com os pacientes. Outros pontos ressaltados pelos discursos dos participantes consistem em fatores que dificultam a assistência continuada à população em situação de rua. (Abreu, 2017)

Pelos discursos apresentados pelos participantes os fatores principais que dificultam a assistência continuada se relacionam com a própria situação de vulnerabilidade dos pacientes e com o déficit de autocuidado, devido às condições que vivem em situação de rua.

Uma dificuldade relatada pelos voluntários é o perfil dos pacientes que são usuários de drogas rejeitam o tratamento, não existe adesão também por estar itinerante então assim dificulta a assistência continuada ao paciente. Fato que os pacientes não compreendem o próprio protagonismo do cuidado causa assim na equipe sentimento de frustração. Nesse sentido, o trabalho com as mulheres em situação de rua é acometido por essas barreiras ressaltadas impedindo assim acompanhamento do pré-natal conforme evidenciado. (Timóteo, et al., 2020)

Portanto, esse déficit de autocuidado dificulta o acesso do paciente afetando assim a atenção à saúde. Nesse sentido, cabe a equipe incentiva e promover meios ao autocuidado desse público por está incluso essa atribuição pelos profissionais, fortalecendo o direito a saúde e sua cidadania, e cada vez mais empoderar o paciente pela busca dos seus direitos. (Balieiro, 2017)

A equipe do consultório de rua deve proporcionar atenção de conforme as necessidades dos pacientes, por lidar por diferentes situações e problemas, que o atendimento deve atender dinâmico. Ainda envolvendo a busca ativa e os cuidados de utilizar dos diversos recursos, meios jurídicos e outras instituições que tenham demanda assim observando o cuidado integral e holístico do paciente. (Rocha, et al., 2021)

\section{Conclusão}

Conforme os resultados apresentados, os discursos dos participantes, que a equipe consultório na rua de Imperatriz atua prestando assistência de acordo com os princípios basilares do Sistema único de Saúde (SUS) e da Política Nacional para a População em Situação de Rua.

Ainda através de meios que colaboram na assistência, incluindo a redução dos danos como estratégia, existe a ação de escuta ativa com o acolhimento, a articulação da rede e nesse aspecto com encaminhamento e garantindo o acesso à rede de atenção à saúde incluindo a RAPS para atenção psicossocial, dessa forma considerando o paciente de forma integral com todas as suas necessidades. E a disponibilidade da equipe que atende em turnos propícios para busca ativa dos pacientes. Notório que os profissionais possuem conhecimento sobre a atuação e finalidade do consultório na rua.

Dentre as dificuldades apontadas a principal o déficit do autocuidado e a rejeição que pela falta do conhecimento sobre os serviços de saúde e de não reconhecer sobre como protagonista dos seus cuidados.

Este estudo apresenta como relevância evidenciar a importância de a equipe manter a articulação com a rede de saúde para prestação de assistência continuada, para que a equipe não seja apenas para os momentos de urgência, mas seja o acompanhamento e a porta de entrada dos serviços de forma efetiva.

Portanto, a formação de fortalecimento das parceiras sejam maiores para que a rejeição não seja barreira para deixar de acompanhar o paciente.

\section{Referências}

Abreu, D. D., \& Oliveira, W. F. D. (2017). Atenção à saúde da população em situação de rua: um desafio para o Consultório na Rua e para o Sistema Único de Saúde. Cadernos de Saúde Pública, 33.

Bardin, Laurence. Análise de Conteúdo. São Paulo: Edições 70 - Brasil, (2016). https://madmunifacs.files.wordpress.com/2016/08/anc3a1lise-decontec3bado-laurence-bardin.pdf. 
Balieiro, E. O., Soares, C. P. D., \& de Araújo Vieira, E. (2017). Morador de rua: causas, entraves e serviços ofertados. Temporalis, 17(34), 333-353.

Bittencourt, M. N., Pantoja, P. V. D. N., Silva, P. C. B. D., Pena, J. L. D. C., Nemer, C. R. B., \& Moreira, R. P. (2019). Consultório na rua: as práticas de cuidado com usuários de álcool e outras drogas em Macapá. Escola Anna Nery, 23.

Brasil. Ministério da Saúde. Portaria N ${ }^{\circ}$ 122, de 25 de janeiro de 2011. Define as diretrizes de organização e funcionamento das Equipes de Consultório na Rua. Diário Oficial da União. (2012). https://bvsms.saude.gov.br/bvs/saudelegis/gm/2012/prt0122_25_01_2012.html .

Brasil. Ministério da Saúde. Secretaria de Atenção à Saúde. Departamento de Atenção Básica. Manual sobre o cuidado à saúde junto a população em situação de rua / Ministério da Saúde. Secretaria de Atenção à Saúde. Departamento de Atenção Básica. - Brasília: Ministério da Saúde. Diário Oficial da União. (2012). http://189.28.128.100/dab/docs/publicacoes/geral/manual_cuidado_populalcao_rua.pdf

BRASIL. Ministério da Saúde. Gabinete do Ministro. Portaria N ${ }^{\circ} 1.255$, De 18 De Junho De 2021. Dispõe sobre as diretrizes de organização e funcionamento das equipes de Consultório na Rua e os critérios de cálculo do número máximo de equipes de Consultório na Rua, por município e Distrito Federal, por meio da alteração da Portaria de Consolidação GM/MS nº 2, de 28 de setembro de 2017. Diário Oficial da União. (2021). https://www.in.gov.br/web/dou/-/portarian-1.255-de-18-de-junho-de-2021-326852389.

BRASIL. Presidência da República. Casa Civil. Subchefia para Assuntos Jurídicos. DECRETO No 7.053 de 23 de dezembro de 2009 . Institui a Política Nacional para a População em Situação de Rua e seu Comitê Intersetorial de Acompanhamento e Monitoramento. Diário Oficial da União. (2009). http://www.planalto.gov.br/ccivil_03/_ato2007-2010/2009/decreto/d7053.htm.

Engstrom, E. M., \& Teixeira, M. B. (2016). Equipe "Consultório na Rua” de Manguinhos, Rio de Janeiro, Brasil: práticas de cuidado e promoção da saúde em um território vulnerável. Ciência \& Saúde Coletiva, 21, 1839-1848.

Ferreira, C. P. D. S., Rozendo, C. A., \& Melo, G. B. D. (2016). Consultório na Rua em Uma Capital do Nordeste brasileiro: o olhar de pessoas em situação de vulnerabilidade social. Cadernos de Saúde Pública, 32, e00070515.

Gil, A. C. (2002). Como elaborar projetos de pesquisa (Vol. 4, p. 175). Atlas.

IBGE - Instituto Brasileiro de Geografia e Estatística. Habitantes de Imperatriz. (2021). Imperatriz (MA)|Cidades e Estados | IBGE

IPEA. Instituto de Pesquisa Econômica Aplicada. Estimativa da População em Situação de Rua no Brasil. Brasília: IPEA (2016). http://repositorio.ipea.gov.br/bitstream/11058/7289/1/td_2246.pdf.

Lozada, G., \& Nunes, K. D. S. (2018). Metodologia científica. SAGAH.

Lima, A. F. S., Almeida, L. W. D. S., Costa, L. D. M. C., Marques, E. S., Lima, M. C. F., \& Rocha, K. R. D. S. L. (2019). Reconhecimento dos riscos no trabalho do Consultório na Rua: um processo participativo. Revista da Escola de Enfermagem da USP, 53.

Oliveira, D. M. D., Expedito, A. C., Aleixo, M. T., Carneiro, N. S., Jesus, M. C. P. D., \& Merighi, M. A. B. (2018). Necessidades, produção do cuidado e expectativas de pessoas em situação de rua. Revista Brasileira de Enfermagem, 71 , 2689-2697. https://www.scielo.br/j/reben/a/dwQXqrrS6KJfHcrCNxmJV9F/abstract/?lang=pt.

Pinto, A. H., Fermo, V. C., Peiter, C. C., Fernandes, V. M. B., \& Heideman, I. T. S. B. (2018). Determinantes sociais, equidade e consultório na rua. Rev. enferm. UFPE on line, 3513-3520.

Santos, F. S., Brito, E. V., de Oliveira, K. C., dos Santos, M. H. D. S., Santos, R. M. D. M. S., Fontoura, I. G., \& Neto, M. S. (2021). Consultório na rua: percepção da equipe multiprofissional sobre a efetividade das ações. Humanidades \& Inovação, 8(45), 59-70.

Rocha Lima, D. G., dos Santos, A. M., \& Martins, S. C. (2021). Invisíveis a céu aberto: gestante em situação de rua. Research, Society and Development, 10(4), e22910414061-e22910414061.

Rosa, A. D. S., Cavicchioli, M. G. S., \& Brêtas, A. C. P. (2005). O processo saúde-doença-cuidado e a população em situação de rua. Revista LatinoAmericana de Enfermagem, 13, 576-582.

Timóteo, A. V. G., dos Santos Silva, J. V., Gomes, L. K. G., Alves, A. S. S., da Silva Barbosa, V. M., \& Brandão, T. M. (2020). Caracterização do trabalho e ações desenvolvidas pelas equipes do Consultório na Rua de Maceió-AL. Enfermagem em Foco, 11(1). 